\title{
A DROWSINESS DETECTION SYSTEM USING COMPUTER VISION AND IoT
}

\author{
Uche M. Chikezie ${ }^{1}$, Nkolika O. Nwazor (Ph.D.)*2 \\ ${ }^{1}$ Department of Electrical/Electronic Engineering, Akanu Ibiam Federal Polytechnic Unwana, 490003, Ebonyi State \\ 2*Department of Electrical/Electronic Engineering, University of Port Harcourt, 500102, Nigeria
}

\begin{abstract}
This paper presents the implementation of a drowsiness detection system using Computer Vision and IoT. The aim is to present an adaptive system that monitors the driver's face and detects when a driver is displaying drowsy signs based on a preset threshold level. This was achieved using algorithms compiled from OpenCV and Dlib libraries, implemented on a Raspberry Pi using Python codes. The implemented system was tested in real-life scenarios and the result showed a drowsy detection accuracy of $90 \%$.
\end{abstract}

Keywords: Open CV; Drowsy driver; Raspberry pi; Internet of Things, Computer Vision

\section{INTRODUCTION}

Driver drowsiness has been the main cause of countless road accidents and this could be due to tiredness, tedious road condition, and unfavorable climate situations (Vural, 2007). As is evident from daily newspapers, news, and social media, accidents are becoming a common trend today. Every year an average of 12,077 road accidents occurs, resulting in about 75400 fatalities (FRSC,2015)These fatalities could include the death of loved ones, such as spouses and wives children, parents, loss of properties, among other things that are negatively affected. Drowsy driving, reckless driving, inattention while driving, lack of proper driving skills, driving too fast, bad roads, system failure on the vehicle, and poor physiological condition have been recognized as some of the causes of road accidents.

Over the years there have been different methods employed to tackle the causes of accidents. These involve the use of administrative procedures such as compulsory use of driver's license for all drivers to ensure that drivers are well trained and licensed, the use of speed control signs (maximum speed limit for various routes), the use of federal road safety commission to ensure compliance with safety rules, motor traffic division among other safety regulations. Some other measures involve applying technological means such as automated brake control system and cruise control system, speed monitor, lane departure warning system among others. Although these measures have achieved success, the rate at which accidents occur incrementally each year reveals that these traditional measures are just not sufficient, therefore there is a need for a system that yields better results. As a result accident prevention and control systems have attracted much research interest recently, intending to propose a one-time solution. According to (Eoh et al, 2005; Daza et al,2011; Dong et al, 2011) the poor physiological state of the driver, causes the majority of these incidents resulting in over $20 \%$ of all car-related accidents.

Drowsy driving is a state where one experiences loss of concentration and focus during driving (Gwak et al, 2018). When a driver is drowsy he experiences attributes such as constant yawing, too much consecutive eye blinking, drooping head, microsleep among other fatigue behaviors while driving. These behaviors are very dangerous if allowed to progress because within seconds accidents can occur and destroy lots of lives and properties.

There are generally three broad categories of drowsy driver detection approaches under which all techniques fall. They are the physiological approach (Hulnhagen,2010) vehicle-based approach (Keyong et al, 2015), and behavioral approach. The approaches have recorded a level of success in trying to solve the problem of drowsiness, however physiological approach (Hulnhagen,2010) is quite invasive and therefore suffers from complexity and interferes with the driving process when installed, as the system is attached to the driver's body to collect data for processing. The vehicle-based approach (Keyong et al, 2015), on the other hand, is meant to respond based on linearity therefore, it has a high rate of false alarms. However various factors other than drowsiness, like bad roads, potholes, etc. can induce nonlinearity on the vehicle. Recent research works aimed at solving the problem of driver drowsiness are based on the behavioral approach (Barr et al, No Date). Behavioral methods employ various techniques like image processing and the use of artificial intelligence. These techniques use real-time images from the drowsy driver dataset to process and predict driver behavior, and if drowsiness is sensed, the system sends a predefined alert. Image processing technique such as color-based approach; segmentation and others examined in (Kumar and Patra, 2018) suffers low performance as a result of lack of intelligence. Hence a better and improved system is proposed with a data-gathering system that collects quality data from the driver and feeds it to the processing center of the system. The processing center used for the proposed system is a Raspberry pi where image processing using OpenCV will be performed. To achieve this improved quality data collection, 


\section{International Advanced Research Journal in Science, Engineering and Technology}

\section{Vol. 8, Issue 12, December 2021}

\section{DOI: $10.17148 /$ IARJSET.2021.81201}

computer vision will be used, while algorithms from pre-trained faces will be used for feature extraction after a face has been detected. OpenCV will be used for real-time face detection, Dlib's pre-trained facial landmark detector will also be used as a complementary library to OpenCV for face recognition and feature extraction. The eye aspect ratio (EAR) is then computed [12] and used to take any action based on the comparison between predefined values and upcoming values of the EAR.

The objectives of the work are to carry out face detection and feature extraction from a video stream using OpenCV and Dlib's pre-trained facial landmark detector, compute the EAR of the extracted feature ( the eyes) to determine if the driver is sleeping, and evaluate the performance of the prototype using real-life scenarios.

\section{MATERIALS AND METHODS}

The proposed system is prototype hardware that will be installed in a vehicle to monitor and analyze the different facial attributes of the driver, with the eyes being the main region of interest to study the state of the driver. Eyeblink is the main focus in detecting the drowsiness of the driver. When the device is turned on, the camera captures a stream of video from which a driver's face is to be detected. Open CV algorithms are used to detect faces in the real-time video stream being collected by the camera. If a face is detected, Dlib's pre-trained facial landmark detector is used to localize facial features and make predictions. The model of the facial landmark localization is presented in equation 1. From equation 1 it was discovered that the eyes aspect ratio(EAR) is approximately constant with wide ajar eyes, and will fall quickly to zero when a blink happens. After the eyes aspect ratio has been computed to determine if the eye is closed, the eye aspect ratio (EAR) threshold can be experimentally determined as illustrated in figure 1.

$\mathrm{EAR}=\frac{\|\mathrm{P} 2-\mathrm{P} 6\|+\|\mathrm{P} 3-\mathrm{P} 5\|}{2\|\mathrm{P} 1-\mathrm{P} 4\|}$

Equation 1

Where $\mathrm{p} 1, \ldots . . \mathrm{p} 6$, are 2D landmark locations as depicted in figure 2

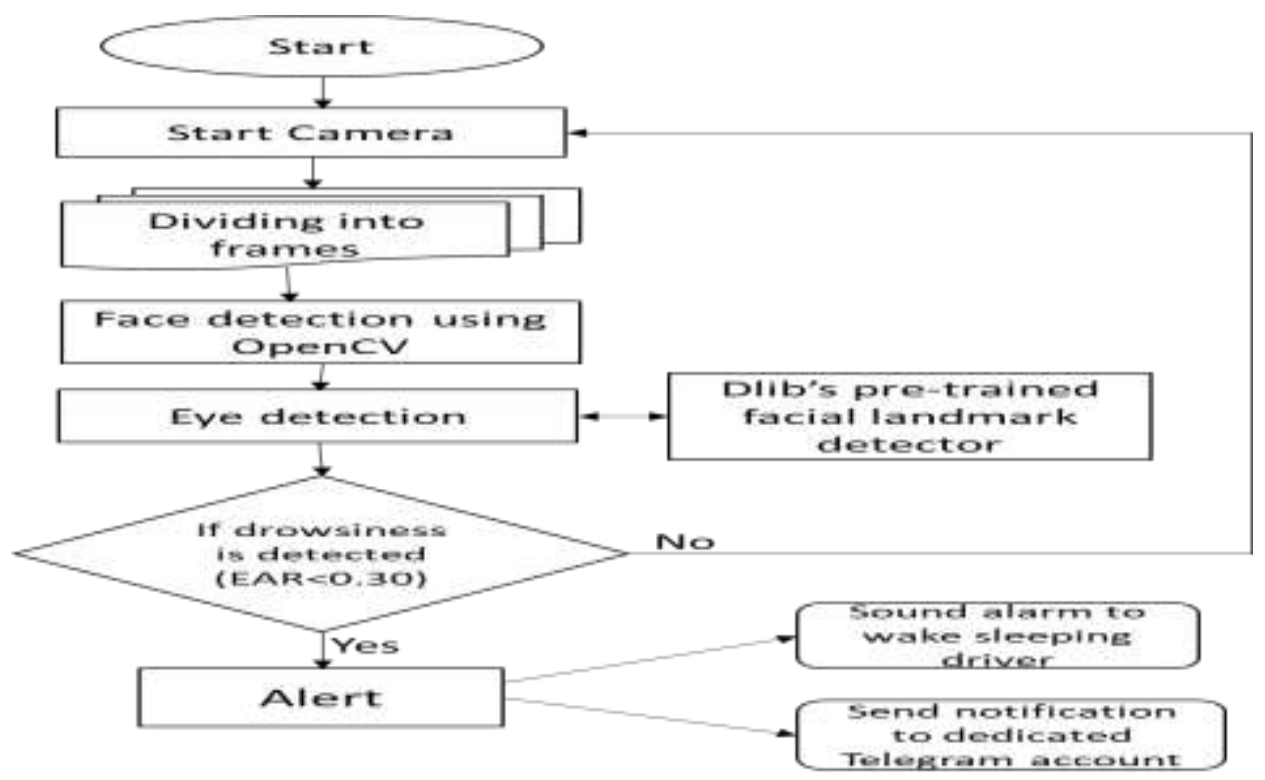

Figure 1: Flow diagram of the Proposed System

The threshold value of an EAR was set at 0.30 for 5 consecutive frames. The number of video frames of the driver's closed eyes is represented by a threshold value of a drowsy eye blink sample. If the determined EAR falls below this predetermined doorstep, the number of frames for which the driver's eyes are closed is counted. If the consecutive number of frames in which the driver have had their eyes closed exceeds the preset threshold number of 5 (indicating that the eye has been closed for a sufficiently long time, an alarm will be sounded which will jerk up the driver and also send an alert to an authorized account on a messaging application.

The described modules work properly through Raspberry Pi model B which is programmed in Python programming language. Python is used because it is the native programming language of Linux which is the host operating system used for this work. It also has very rich libraries for image processing and machine learning. It is relatively easier to code with Python and maintain the code. The command to carry out the IoT notification of the sleepy driver is also embedded in the python codes. If the consecutive counting frames increase above the range of the threshold value set for the limit at which the eyes can be closed, then drowsiness of the driver is detected. 
Vol. 8, Issue 12, December 2021

DOI: $10.17148 /$ IARJSET.2021.81201
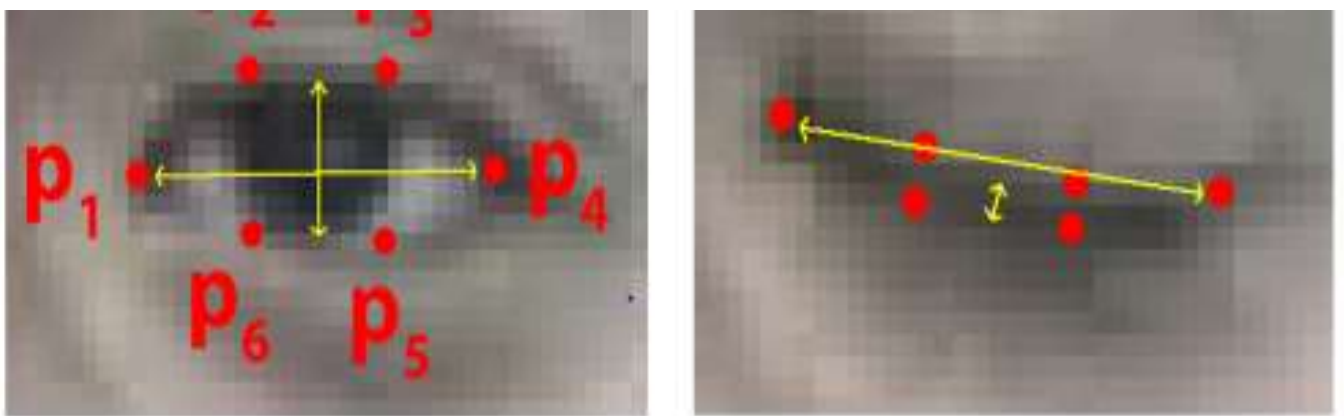

Figure 2: Important coordinates/eye landmarks used to determine EAR.

\section{A. System Operation}

The flowchart in figure 3 shows the operation of the prototype hardware. If the camera is properly connected, system initialization takes place to ensure that the Raspberry Pi is connected to the internet and also to the registered Telegram account. The system then captures a video stream and scans it for the presence of a human face. If no face is found it repeats the process of capturing a video and scanning for a face till a face is found. Data containing different drowsy behaviors are captured and sent to the Raspberry Pi for processing. The video data is composed of image frames synchronized in 30 frames per second. When a face is found it scans it for the Eye Aspect Ratio (EAR) and checks if the EAR is below a pre-set threshold level. If it is above it continues scanning for the EAR until it becomes lower than the threshold value for more than 5 consecutive frames, then it can go ahead to sound an alarm warning, captures the image of the sleepy driver, and sends it to a dedicated telegram account as long as the internet is still connected.

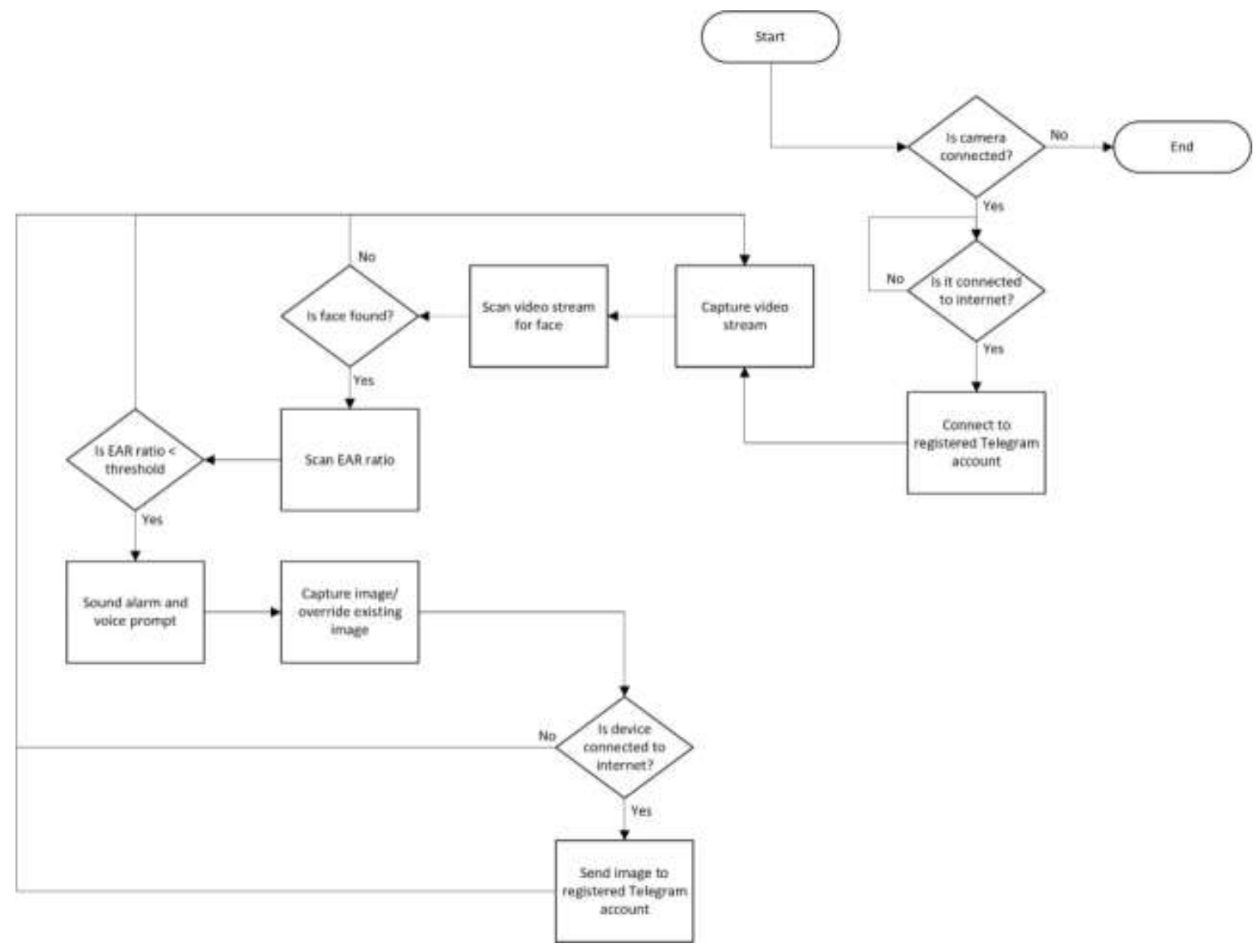

Figure 3: System Operation 
International Advanced Research Journal in Science, Engineering and Technology

Vol. 8, Issue 12, December 2021

DOI: $10.17148 /$ IARJSET.2021.81201

\section{RESULTS AND DISCUSSION}

The Raspberry Pi was integrated with the camera, internet dongle, Infra-red light, speakers, and remote telegram account. The program for the Raspberry pi was written with a python script. Figures 4 and 5, show the implemented hardware design.

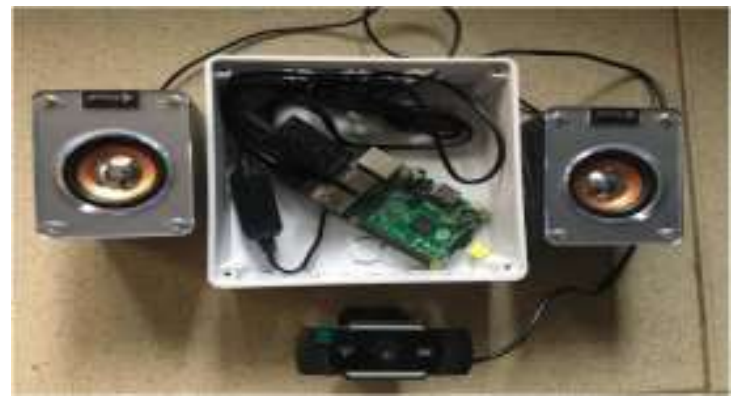

Figure 4: Assembled Prototype

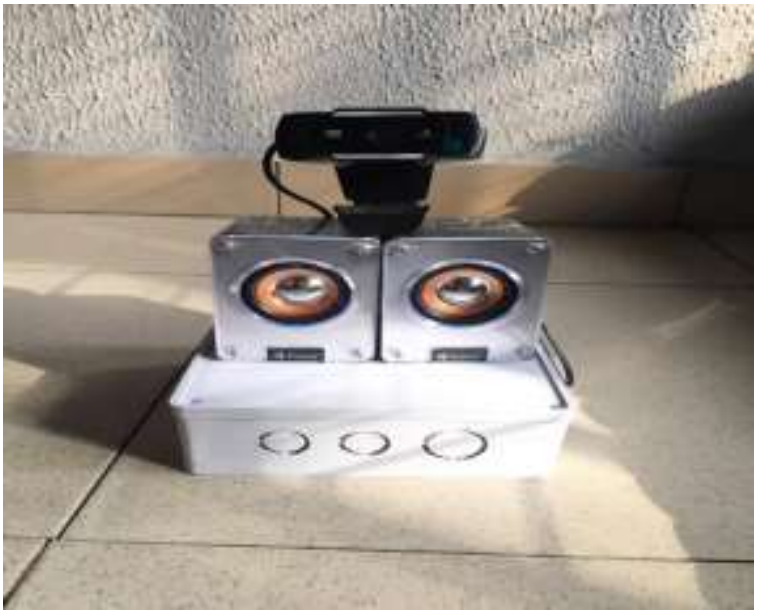

Figure 5: Coupled Prototype

The hardware prototype was installed on a Ford Edge as shown in figure 6 . As shown in figure 6 the camera is positioned on the rearview mirror to take the user's behavior on the go and part of the hardware is placed in a temporal make-shift space in the car.

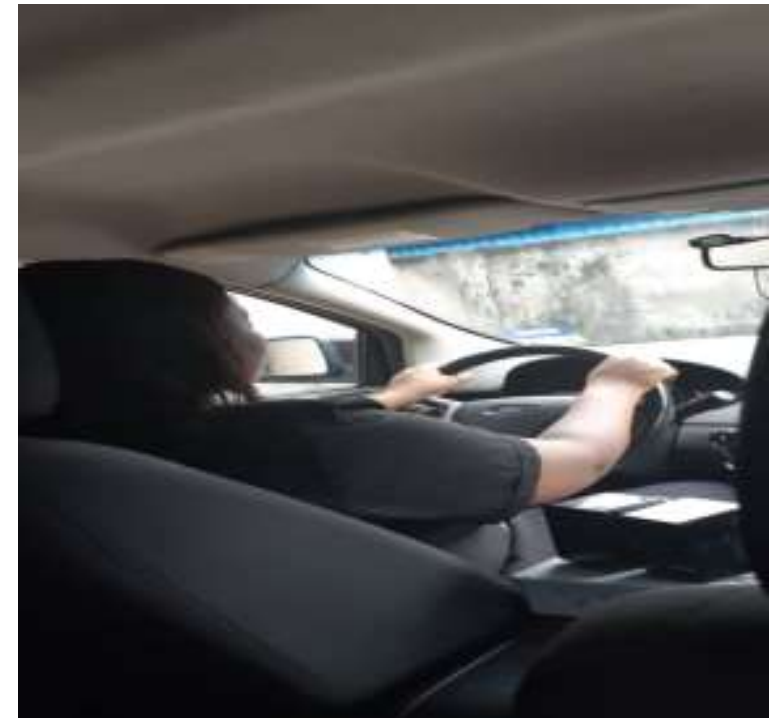

Figure 6: Prototype hardware installation 
Vol. 8, Issue 12, December 2021

DOI: 10.17148/IARJSET.2021.81201

The performance of the hardware was then tested with different behaviors and the results are shown in Table 1 . This test was done with the driver sleeping for different durations. This was done ten times and had 1 false negative. For the true positive results an alarm sounded and the warning "WAKE UP" is voiced out. The results are as arranged in table 1. From table 1, it is seen that out of 10 tests carried out 1 wasn't able to detect drowsiness/drowsy conditions thereby keeping the accuracy of the system at $90 \%$.

Table 4.3: Results of the Prototype Hardware Testing

\begin{tabular}{|l|l|l|}
\hline $\begin{array}{l}\text { Driver with different } \\
\text { Driving Behaviors }\end{array}$ & Alarm & $\begin{array}{l}\text { Telegram } \\
\text { Picture }\end{array}$ \\
\hline 1 & Yes & Yes \\
\hline 2 & Yes & Yes \\
\hline 3 & Yes & Yes \\
\hline 4 & Yes & Yes \\
\hline 5 & Yes & Yes \\
\hline 6 & Yes & Yes \\
\hline 7 & No & No \\
\hline 8 & Yes & Yes \\
\hline 9 & Yes & Yes \\
\hline 10 & Yes & Yes \\
\hline
\end{tabular}

In addition to the wake-up alert, images of the drowsy driver are taken and sent to a designated Telegram account (a messaging app) which serves as the admin or control terminal to serve as feedback and to monitor the driver's state. Some images of the driver at different EARs and driving behaviors are shown in figures 7 and 8 . Figure 9. Shows an alert notification that was taken by the system and sent to the telegram account.

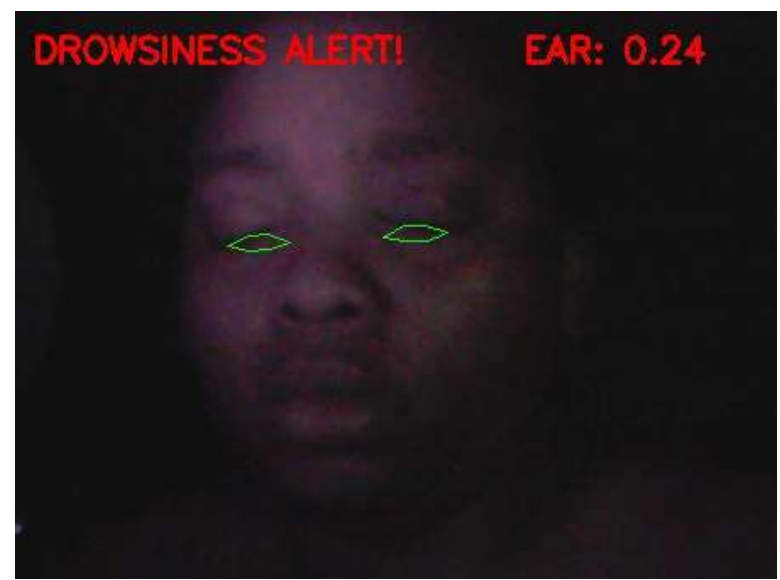

Figure 7: Driver Image captured by the system with an EAR: 0.24

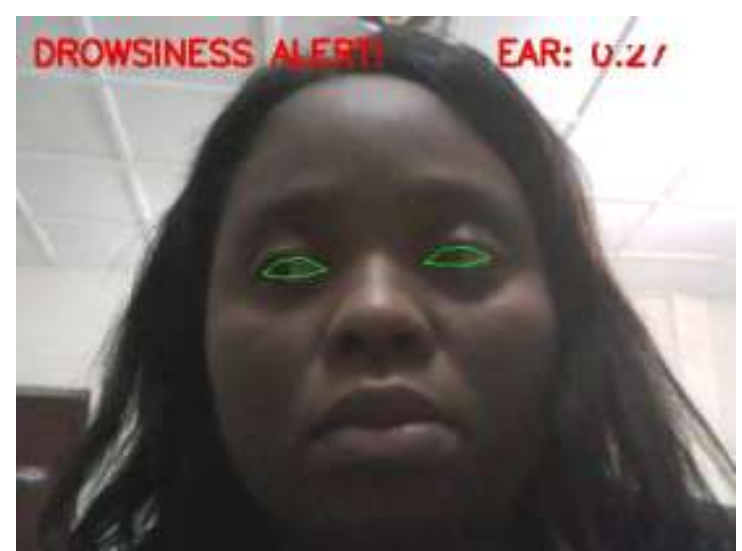

Figure 8: Driver Image captured by the system with an $\mathrm{EAR}=\mathbf{0 . 2 7}$ 


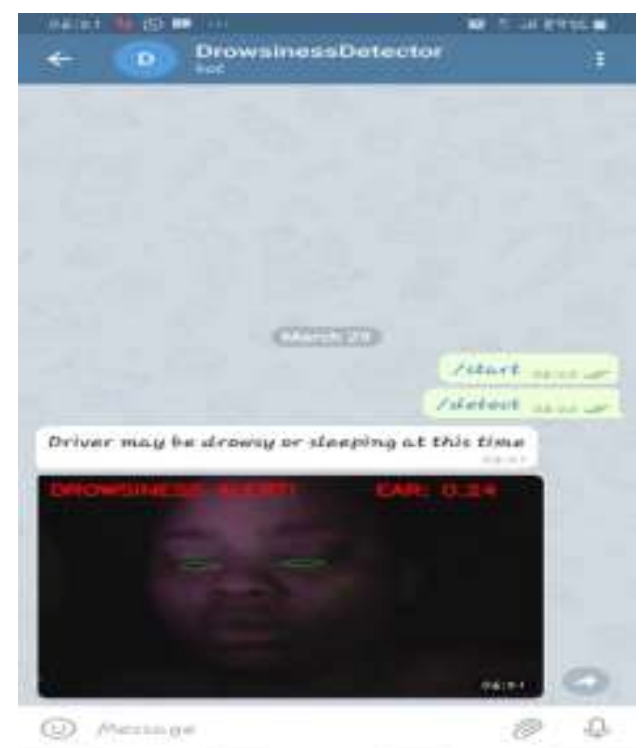

Figure 9: Alert notification received on telegram messaging app

\section{CONCLUSION}

This research has successfully developed a drowsiness detection system that monitors the state of the driver in real-time and gives an alert both as an alarm and a remote notification to a messaging app. A behavioral-based approach was adopted because the existing techniques based on psychological and vehicle-based methods are intrusive and unreliable. A hardware prototype to check the drowsiness of the driver and send a notification via IoT was developed. The system's main components are the Raspberry Pi3 model B module, USB webcam camera used for real-time capturing of data that contains of a stream of video on which face detection is performed using OpenCV. Facial landmarks localization was performed using Dlib's pre-trained facial landmark detector and then the localized facial landmark points were used to calculate EAR. If the calculated EAR value increases from the preset threshold, it means the eyes are still open and no change in the state of the system occurs. Similarly, if the EAR value falls below the threshold, then the system urgently alerts the driver using a voice note and a warning message containing an image of the sleeping is also sent to a telegram account driver that serves as admin/ the regulatory body of the organization. The system was tested and seen to have an accuracy of $90 \%$.

\section{REFERENCES}

Vural, Esra \& Cetin, Mujdat \& Ercil, Aytul \& Littlewort, Gwen \& Movellan, Javier. (2007). Drowsy Driver Detection Through Facial Movement Analysis. IEEE International Workshop on Human-Computer Interaction, Berlin, Heidelberg: Springer Berlin Heidelberg: 6-18. DOI:10.1007/978-3-540-75773-3 2

Boboye Oyeyemi (2015). FRSC Statistical Digest, $1^{\text {st }}$ Quarter, 2015 Edition. https://frsc.gov.ng/statdigest1.pdf Accessed $15^{\text {th }}$ July, 2021

Eoh, H. J., Chung, M. K., \& Kim, S. H. (2005). Electroencephalographic study of drowsiness in simulated driving with sleep deprivation. International Journal of Industrial Ergonomics, 35(4), 307--320. https://doi.org/10.1016/i.ergon.2004.09.006

Daza, I.G.; Hernandez, N.; Bergasa, L.M.; Parra, I.; Yebes, J.J.; Gavilan, M.; Quintero, R.; Llorca, D.F.; Sotelo, M.A.(2011). Drowsiness monitoring based on driver and driving data fusion. In Proceedings of the 14th international IEEE conference on intelligent transportation systems (ITSC), Washington, DC, USA, 5-7 October 2011:11991204. https://ivan-garcia.uah.es/static/conference_papers/Drowsiness_monitoring_based_ITSC2011.pdf

Dong, Y., Hu, Z., Uchimura, K., and Murayama, N. (2011). Driver inattention monitoring system for intelligent vehicles: A review, IEEE Transactions on Intelligent Transportation Systems. 12 (2): 596-614. DOI: 10.1109/TITS.2010.2092770

Gwak J, Hirao A, Shino M.(2020). An Investigation of Early Detection of Driver Drowsiness Using Ensemble Machine Learning Based on Hybrid Sensing. Applied Sciences. 2020; 10(8):2890. https://doi.org/10.3390/app10082890

Hulnhagen T, Dengler I, Tamke A, Dang T and Breuel G (2010). Maneuver recognition using probabilistic finite-state machines and fuzzy logic. IEEE Intelligent Vehicles $\begin{array}{llll}\text { Symposium. IEEE. San Diego USA, 2010, } & \text { Une, }\end{array}$ https://www.researchgate.net/publication/281522219_Observing_Behaviors_at_Intersections_A_Review_of_Recent_Studies_Developments

Keyong L, Lisheng J, Yuying. J, Huacai. X, Linlin G. Effects of driver behavior style differences and individual differences on driver sleepiness detection. Adv Mech Eng [Internet]. 2015; Available from: http://dx.doi.org/1687814015578354.

Barr, L.; Howarth, H.; Popkin, S.; Carroll, R. J.(No Date) A review and evaluation of emerging driver fatigue detection. John A. Volpe National Transportation Systems Center Cambridge, Massachusetts http://citeseerx.ist.psu.edu/viewdoc/download?doi=10.1.1.508.8409\&rep=rep1\&type=pdf

Kumar A and Patra R.(2018). Driver drowsiness monitoring system using visual behavior and machine learning. IEEE Symposium on Computer Applications \& Industrial Electronics (ISCAIE). 339-344. DOI: 10.1109/ISCAIE.2018.8405495

Biswal AK, Singh D, Pattanayak BK, Samanta D and Yang M-H (2021). IoT-based smart alert system for drowsy driver detection. Wirel Commun Mob Comput :1-13. 